\title{
MATHEMATICAL EXPRESSION OF THE FORM OF A HELIO CONCENTRATOR
}

\author{
Vitali Severyanin ${ }^{1, *}$, Pavel Yanchilin ${ }^{1}$ \\ ${ }^{1}$ Brest State Technical University, Faculty of Engineering systems and Ecology, Maskouskaya str. 267, \\ 224017, Brest, Republic of Belarus
}

\begin{abstract}
It is necessary to increase the efficiency of existing solar power plants both through the study of energy processes and the creation of new schematic diagrams of solar systems by reducing the cost of their design, principle of operation and operation. Authors have developed the LUCH solar plant in solving these problems. The article presents the device of the installation, a mathematical description of the shape of the solar concentrator, calculations of the parameters of the cones and their layout diagram. When developing the concentrator, the condition was accepted the adopted form of the paraboloid as the basis of the concentrator needs to be changed so as to simplify the design and manufacturing process. The design change was made by splitting the paraboloid of revolution into separate cones. The calculated geometrical parameters of the LUCH solar concentrator are compared with the obtained parameters for an ideal paraboloid concentrator.

Keywords: solar power plants, solar radiation concentrators, solar radiation flux intensity, paraboloid, mathematical description, splitting of a paraboloid of rotation.
\end{abstract}

\section{Introduction}

The growing demand for energy — electricity and heat - forces, despite many years of large-scale research, development and application, to look for further ways to develop solar plants. To increase the efficiency of existing solar power plants complex designs and expensive materials are used which increases the payback period of solar power plants. A serious obstacle to the effective implementation of the high energy potential of solar radiation is its low density because of the great distance between the Earth and the Sun. This contradiction can only be overcome by concentrating radiation.

The long history of the development of energy-saving equipment in Europe has allowed accumulating significant experience in this area. It is possible find in the literature a lot of data on the successful implementation of projects of energy efficient systems using renewable energy sources in particular solar using concentrators [1-5].

Authors of the article have developed a solar plant "LUCH" in the research laboratory "PULSAR" of the Brest State Technical University. It refers to municipal industrial heat power engineering and can be used for heating and hot water supply systems for small con-

\footnotetext{
*Corresponding author: tgv_bstu@tut.by
} 
sumers (cottages, greenhouses, workshop premises, warehouses, various technological needs in agriculture) as a backup for fuel-using systems. The purpose of the solar plant is to capture and concentrate the sun's rays in focus on a spherical heat receiver, transfer the heat generated in the focus to the coolant and collect the heated coolant in the storage tank for subsequent consumption.

\section{METHOD}

\subsection{Device Description}

The solar plant design consists of five main parts (Figure 1):

1. Optical system (a complex of cones-mirrors on a special frame - a solar concentrator);

2. Sun tracking system (copiers, optical system turning mechanism, electric drive, control system);

3. Mechanism for lifting the optical system (levers and rods);

4. Heat receiver (spherical solar water heater, pipe-wire system, storage tank, regulators and valves);

5. The solar plant is mounted on a metal base (plate, channels, bearings).

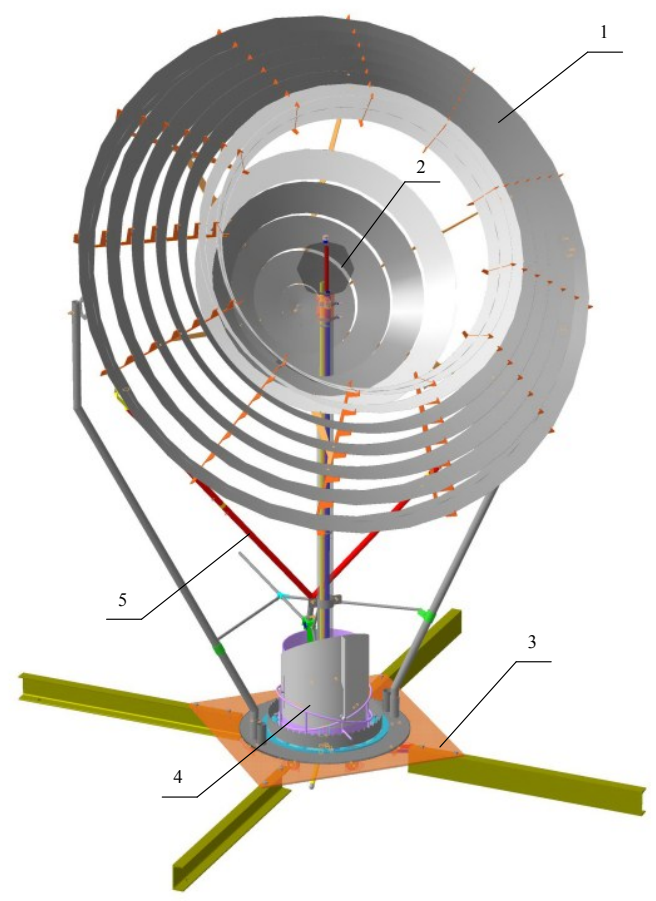

1 - optical system; 2 - heat receiver; 3 - base; 4 - solar tracking system;

5 - lifting mechanism of the optical system.

Figure 1 - Model of a solar plant

The main features (differences from known installations) of the LUCH solar plant are:

- "Splitting" of the continuous rotation of the paraboloid into separate concentric cones and construction from them of a new, more technological, solar concentrator; 
- the original design of the cone solar energy concentrator allows its use in the springautumn and winter periods;

- the use of this concentrator of solar energy can significantly increase the utilization rate of solar energy, as a result of which the temperature of the coolant can be increased to $300^{\circ} \mathrm{C}$;

- mirror part of the solar concentrator is a group of narrow, separated from each other concentric cones which reduces the wind load because the air flow passes through the distance between the cones and simplifies the manufacture and assembly of mirrors since the surfaces of the cones have I degree of curvature;

- the original design of the heat receiver allows you to effectively use concentrated solar energy and the presence of an air gap between the surface of the heat receiver and the transparent shell eliminates heat losses in a convective way;

- the heat receiver is stationary, the optical system rotates around it, this simplifies the design of the coolant communications;

- orientation of the solar concentrator axis to the Sun is carried out by a special tracking mechanism;

- movement of the optical system takes into account not only daily but also seasonal changes in the position of the Sun.

\subsection{Research progress}

When developing the concentrator, the condition was accepted that we take the form of a paraboloid as the basis of the concentrator and it is required to change it so as to simplify the design, manufacturing and operation process. In the course of the geometric transformations shown in Figure 2, we get a new type of concentrator, shown in Figure 3.

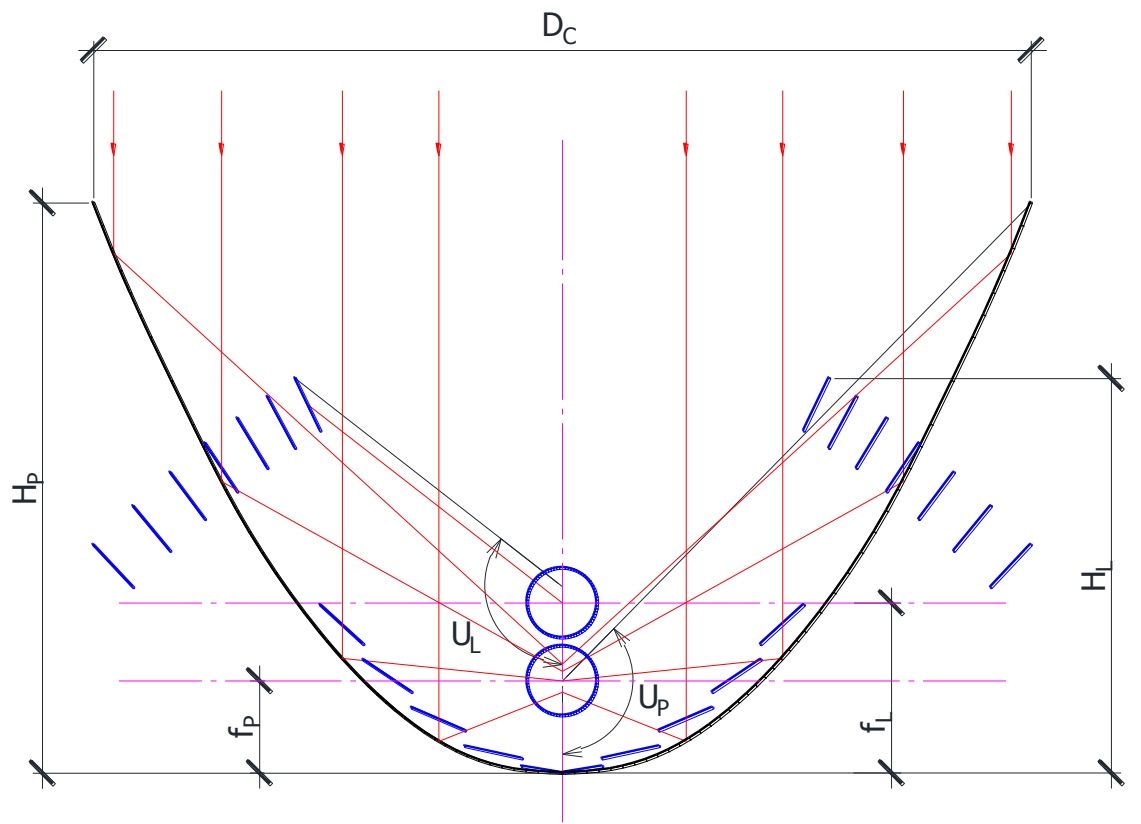

Figure 2 - Combination of two concentrators and their main dimensions

Using a graphical-analytical method based on geometric constructions we will display rays falling and reflected by a mirror. In this case, we use the law of specular reflection and 
we will consider a parallel beam of rays, the distribution of radiation in the beam along the directions is assumed to be uniform. We will simplify the construction by splitting the paraboloid of revolution into separate cones. We get that the concentrator is a collection of concentric cones. Each cone is a tape of sheet material bent in the form of a truncated cone, the inner surface of which is mirrored. The angle of the generatrix of the cone to its axis is chosen so that the sunbeam after reflection is directed into focus. All cones have the same focus.

The optical system or concentrator of the LUCH solar plant consists of three main elements: radii, cones, and fastening rings. Their design dimensions are determined by geometric constructions. The cones are spaced relative to each other by radii, thus forming a blown-through robust spatial structure with less windage. Radii are flat elements that have specially cut shapes for attaching the cones at the required predetermined angle, which is a prerequisite for the best reception of the flow of sunlight. The radii are fixed on support rings that are attached to the posts.

Concentrators in which the generatrix of the reflecting surface is a curve of the second order - a circle, a parabola, a hyperbola, etc. belong to the group of highly concentrated systems. An ideal paraboloid concentrator focuses a parallel beam of rays into a point, which corresponds to an infinitely high degree of concentration $\left(K_{c} \rightarrow \infty\right)$. The geometry of a paraboloid reflector is characterized by two independent parameters: the concentrator diameter $D_{c}$ (or focal length $f_{c}$ ) and the half-opening angle $U_{c}$ (or the $D_{c} / f_{c}$ ratio) [6]. The third parameter is always dependent and is determined from the equation:

$$
D_{C}=4 \cdot f_{C} \cdot \sin U_{C} / 1+\cos U_{C}
$$

These parameters are shown in Figure 2 and differ only in indices: $\boldsymbol{P}$ - for the paraboloid concentrator, $\boldsymbol{L}$ - for the "LUCH" concentrator.

These geometric transformations of the paraboloid concentrator were carried out in order to simplify the design for the manufacturing process and operation. Therefore, it is necessary to compare the geometric characteristics of these two concentrators. An important parameter will be the ratio of the area of the reflecting surface of the concentrator (mirror) $S_{\text {sur }}$ to the area of the captured solar flux $S_{\text {sol }}$ :

$$
K_{x}=S_{\text {sur }} / S_{\text {sol }}
$$

We will use the well-known parabola formulas to calculate the main parameters of the paraboloid concentrator and the area of the reflecting surface of the concentrator (mirror) $S_{\text {sur. }}$. The canonical equation of a parabola in a rectangular coordinate system is:

$$
y^{2}=2 p x, p>0
$$

where $p$ is the focal parameter of the parabola, it is equal to the distance from the focus to the directrix.

Since each point of the parabola is equidistant from the focus and the directrix, so is the vertex, so it lies between the focus and the directrix at a distance $p / 2$ from both. Hence the focal length $f_{c}$, m:

$$
f_{K}=p / 2
$$

The height of the parabola $h$ is found from the ratio:

$$
h=R^{2} / 2 p
$$

where $R$ is the radius of the circle described by the branches of the parabola when rotating around the $Y$-axis (paraboloid), in the plane perpendicular to this axis at a distance $h$ from the apex of the parabola, $m$.

The surface area of the paraboloid or the area of the reflecting surface of the concentrator (mirror) $S_{\text {sur }}$. can be found from the equation:

$$
S_{\text {sur }}=2 \pi \cdot\left(\sqrt{\left(p^{2}+R^{2}\right)^{3}}-p^{3}\right) / 3 p
$$


Let us pose the problem of finding such a parameter of a parabola (and, accordingly, a paraboloid) $\mathrm{p}$ at which its surface area $S_{\text {sur }}$ tends to a minimum. To do this, you need to take the derivative of equation (6) and determine the unconditional maximum of the function, equating it to zero.

$$
\begin{gathered}
S_{\text {sur }}^{\prime}(p)=\frac{2 \pi \cdot\left[3 p \sqrt{\left(p^{2}+R^{2}\right)^{3}}-3 p^{2}-\frac{\left(\sqrt{\left(p^{2}+R^{2}\right)^{3}}-p^{3}\right)}{p}\right] / 3 p=}{3 p}=2 \pi \cdot\left[\sqrt{p^{2}+R^{2}}\left(2 p-\frac{R^{2}}{p}\right)-2 p^{2}\right] / 3 p=0
\end{gathered}
$$

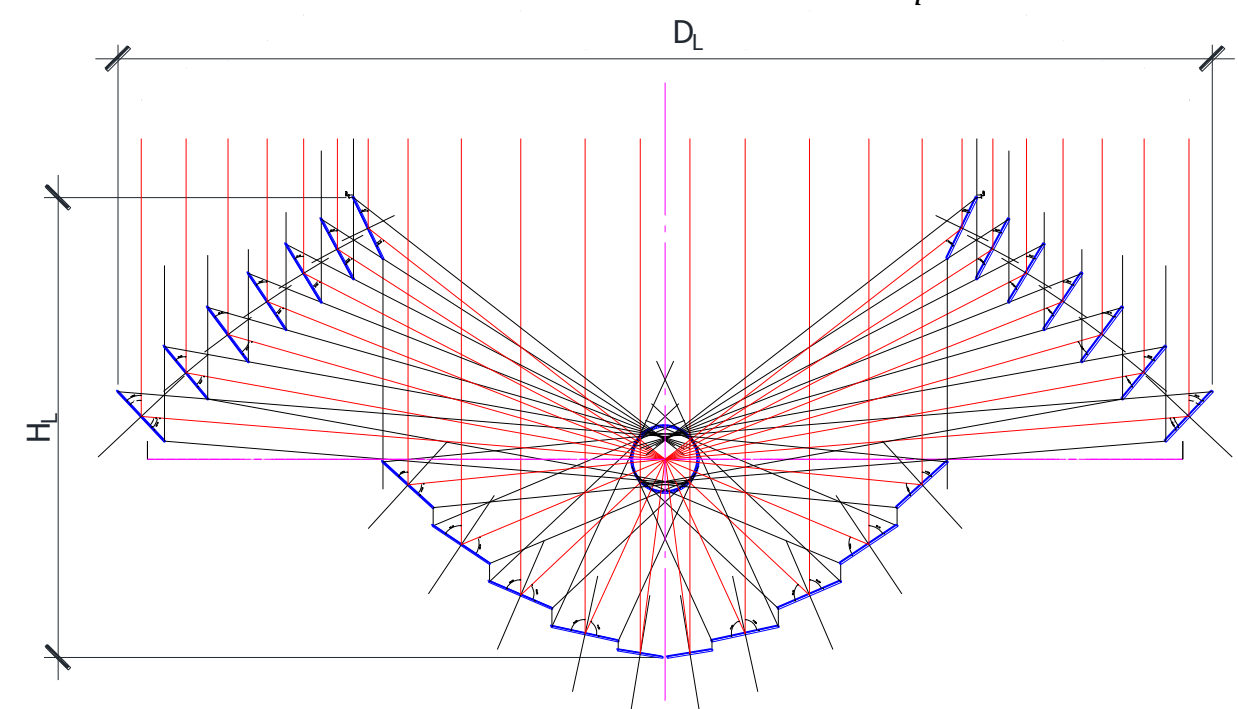

Figure 3 - Concentrator in the form of a split paraboloid

The root of this equation turns out to be $R=0$. Therefore, it is necessary to look for a conditional (local) extremum, for this we accept the constraints established experimentally. As the radius $R$ and the parameter $p$ are related by the relation (5), then we will set the range of heights from 0.1 to 2 meters with a step of $0.01 \mathrm{~m}$. This gap is due to the fact that the calculated ideal paraboloid should correspond as much as possible to the geometric dimensions of our composite concentrator and the height $h_{L}$ of the calculated "LUCH" solar concentrator is $1.016 \mathrm{~m}$. We set the value of the paraboloid radius $R_{P}=1.2 \mathrm{~m}$ which corresponds to the radius of the solar concentrator "LUCH" and, accordingly, the area of the captured solar flux $S_{s o l}=4.52 \mathrm{~m}^{2}$.

\section{RESULTS AND DISCUSSION}

Calculation sequence:

- find the area $S_{\text {sur }}$ from formula (7),

- parameter $p$ from (5),

- focal length $f$ from (4).

Table 1 shows some of the data obtained.

Table 1. Results of calculating the paraboloid

\begin{tabular}{|c|c|c|c|c|}
\hline$S_{\text {sur }}, \mathbf{M}^{2}$ & $h_{P}, \mathbf{M}$ & $p$ & $f, \mathbf{M}$ & $K_{x}$ \\
\hline
\end{tabular}




\begin{tabular}{|c|c|c|c|c|}
\hline 4,56 & 0,1 & 7,2 & 3,6 & 1,01 \\
\hline 4,59 & 0,15 & 4,8 & 2,4 & 1,02 \\
\hline 4,63 & 0,2 & 3,6 & 1,8 & 1,03 \\
\hline 4,71 & 0,25 & 2,88 & 1,44 & 1,04 \\
\hline 4,79 & 0,3 & 2,4 & 1,2 & 1,06 \\
\hline 4,89 & 0,35 & 2,06 & 1,03 & 1,08 \\
\hline 4,99 & 0,4 & 1,8 & 0,9 & 1,10 \\
\hline 5,11 & 0,45 & 1,6 & 0,8 & 1,13 \\
\hline 5,24 & 0,5 & 1,44 & 0,72 & 1,16 \\
\hline 5,37 & 0,55 & 1,31 & 0,65 & 1,19 \\
\hline 5,51 & 0,6 & 1,2 & 0,6 & 1,22 \\
\hline 5,66 & 0,65 & 1,11 & 0,55 & 1,25 \\
\hline 5,82 & 0,7 & 1,03 & 0,51 & 1,29 \\
\hline 5,99 & 0,75 & 0,96 & 0,48 & 1,32 \\
\hline 6,16 & 0,8 & 0,9 & 0,45 & 1,36 \\
\hline 6,19 & 0,81 & 0,89 & 0,44 & 1,37 \\
\hline 6,23 & 0,82 & 0,88 & $0,44=f_{L}$ & 1,38 \\
\hline 6,26 & 0,83 & 0,87 & 0,43 & 1,38 \\
\hline 6,29 & 0,84 & 0,86 & 0,43 & 1,39 \\
\hline 6,33 & 0,85 & 0,85 & 0,42 & 1,40 \\
\hline 6,51 & 0,9 & 0,8 & 0,4 & 1,44 \\
\hline 6,69 & 0,95 & 0,76 & 0,37 & 1,48 \\
\hline 6,89 & 1 & 0,72 & 0,36 & 1,52 \\
\hline 6,92 & 1,01 & 0,71 & 0,35 & 1,53 \\
\hline 6,96 & $1,02=h_{L}$ & 0,71 & 0,35 & 1,54 \\
\hline 7,00 & 1,03 & 0,70 & 0,34 & 1,55 \\
\hline 7,04 & 1,04 & 0,69 & 0,34 & 1,56 \\
\hline 7,08 & 1,05 & 0,68 & 0,34 & 1,57 \\
\hline 7,27 & 1,1 & 0,66 & 0,33 & 1,61 \\
\hline 7,31 & 1,11 & 0,65 & 0,32 & 1,62 \\
\hline $7,35=S_{L}$ & 1,12 & 0,64 & 0,32 & $1,63=\boldsymbol{K}_{\boldsymbol{L}}$ \\
\hline 7,39 & 1,13 & 0,64 & 0,32 & 1,63 \\
\hline 7,43 & 1,14 & 0,634 & 0,31 & 1,64 \\
\hline 7,47 & 1,15 & 0,624 & 0,31 & 1,65 \\
\hline 7,68 & 1,2 & 0,6 & 0,3 & 1,70 \\
\hline 7,88 & 1,25 & 0,58 & 0,29 & 1,74 \\
\hline 8,09 & 1,3 & 0,55 & 0,28 & 1,79 \\
\hline 8,51 & 1,4 & 0,51 & 0,26 & 1,88 \\
\hline 8,94 & 1,5 & 0,48 & 0,24 & 1,98 \\
\hline 9,37 & 1,6 & 0,45 & 0,23 & 2,07 \\
\hline 9,81 & 1,7 & 0,42 & 0,21 & 2,17 \\
\hline 10,26 & 1,8 & 0,4 & 0,2 & 2,27 \\
\hline 10,71 & 1,9 & 0,38 & 0,19 & 2,37 \\
\hline 11,17 & 2 & 0,36 & 0,18 & 2,45 \\
\hline
\end{tabular}

Analyzing the data obtained, it can be seen that the minimum surface area is achieved at the minimum height of the paraboloid, which can be seen from the graph in Figure 4. With an increase in the height $h$, the surface area increases and the focal distance decreases which can be seen from the graph in Figure 5. It is obvious that with an increase the surface area increases and the coefficient $K_{x}$ (graph in Figure 6). 


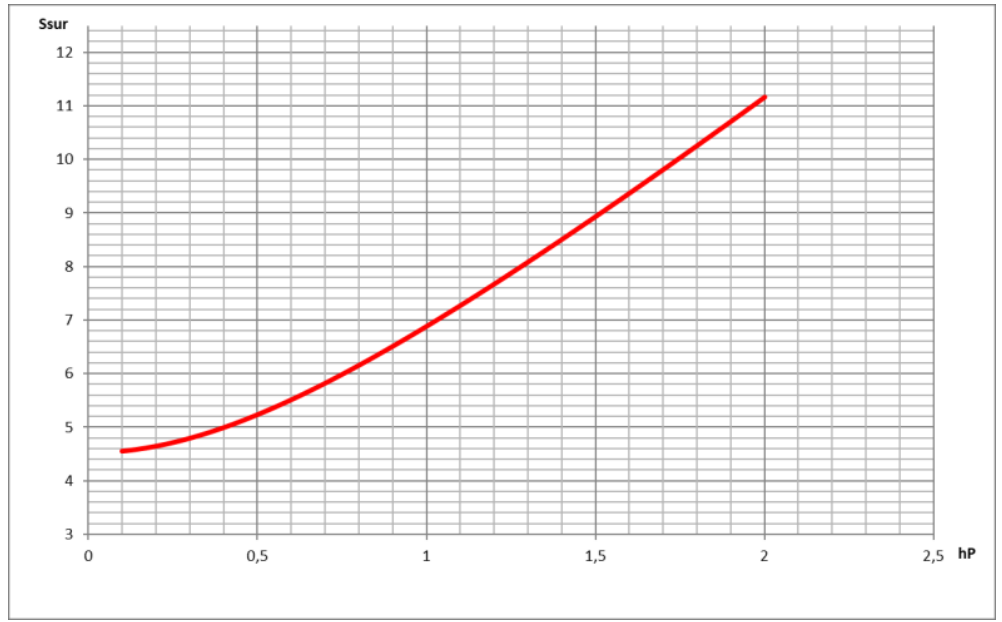

Figure 4 - Graph of dependence of $S_{\text {sur }}$ from $h_{p}$

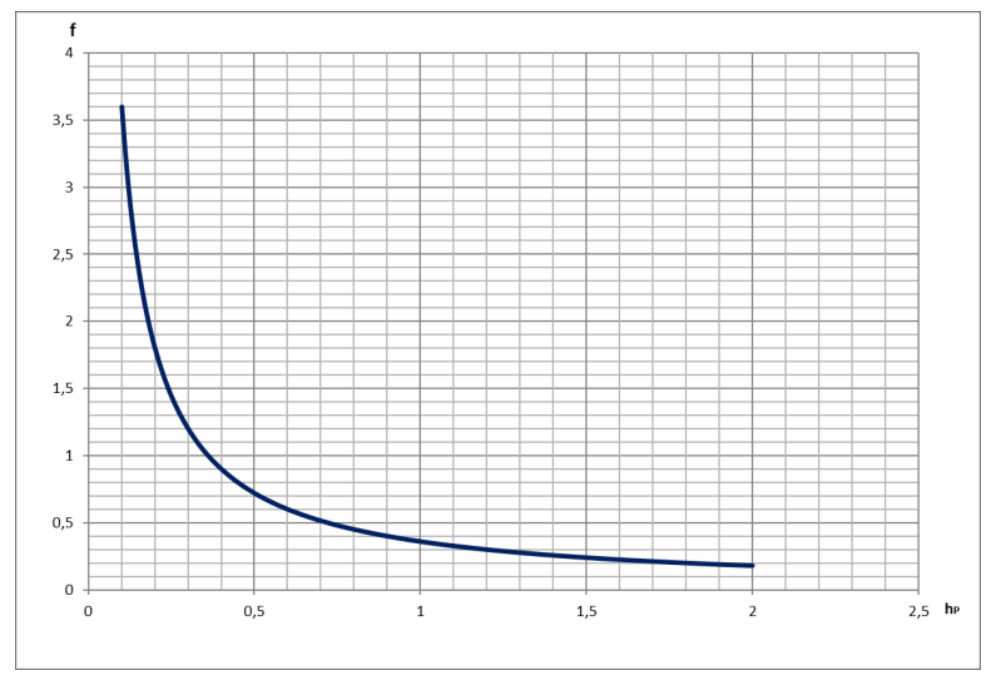

Figure 5 - Graph of $f$ from $h_{p}$

As the radius of the paraboloid and the "LUCH" solar concentrator are equal, then the area of the captured solar flux $S_{\text {sol }}$ is the same for these two concentrators. This means that the heat power $Q$ received, reflected, concentrated and "utilized" (transferred to the heat carrier in the heat receiver) is also the same for them. From an economic point of view, it is unprofitable to produce a concentrator with larger geometrical dimensions, if its thermal power corresponds to a concentrator with smaller dimensions. Therefore, it is necessary to take into account the relationship between the geometric parameters of the concentrator $(R, h, f)$ for a given thermal power of the solar plant. 


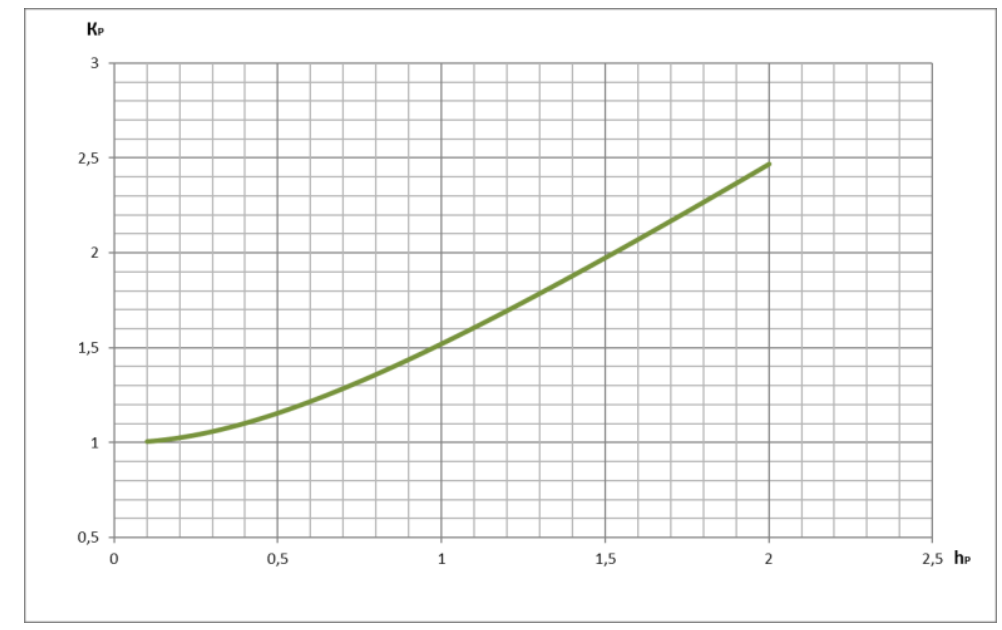

Figure 6 - Graph of dependence of $K p$ on $h p$

Let us compare the geometric parameters of the LUCH solar concentrator calculated earlier by the graphical analytical method with the parameters obtained for the paraboloid concentrator (Table 1, highlighted lines).

Calculated geometrical parameters of the LUCH solar concentrator (from table 2):

- $\quad$ Total height $h=1.02 \mathrm{~m}$;

- $\quad$ Maximum diameter $D=2.4 \mathrm{~m}$, radius $R=1.2 \mathrm{~m}$;

- Focal length $f=0.44 \mathrm{~m}$;

- Surface area of mirrors $S_{\text {sur }}=7.36 \mathrm{~m}^{2}$;

- Coefficient $K_{x}=1.63$.

Calculated geometrical parameters of the paraboloid concentrator at the same height $h$ :

- $\quad$ Total height $h=1.02 \mathrm{~m}$;

- Maximum diameter $D=2.4 \mathrm{~m}$, radius $R=1.2 \mathrm{~m}$;

- $\quad$ Focal length $\mathrm{f}=0.35 \mathrm{~m}$;

- Surface area of the mirror $S_{\text {sur }}=6.96 \mathrm{~m}^{2}$;

- Coefficient $K_{x}=1.54$.

Calculated geometrical parameters of the paraboloid concentrator at the same focus $f$ :

- $\quad$ Total height $h=0.82 \mathrm{~m}$;

- Maximum diameter $D=2.4 \mathrm{~m}$, radius $R=1.2 \mathrm{~m}$;

- Focal length $f=0.44 \mathrm{~m}$;

- $\quad$ Surface area of the mirror $\mathrm{S}_{\text {sur }}=6.23 \mathrm{~m}^{2}$;

- Coefficient $K_{x}=1.38$.

Calculated geometric parameters of the paraboloid concentrator for the same mirror surface area $S_{\text {sur }}$ and the same $K_{x}$ coefficient:

- Total height $h=1.12 \mathrm{~m}$;

- Maximum diameter $D=2.4 \mathrm{~m}$, radius $R=1.2 \mathrm{~m}$;

- Focal length $f=0.32 \mathrm{~m}$;

- Surface area of the mirror $S_{\text {sur }}=7.35 \mathrm{~m}^{2}$;

- Coefficient $K_{x}=1.63$.

The cone of the optical system is shown externally in Figure 7. Its main parameters are the diameter of the larger $\operatorname{arc} D_{\max }$ and the diameter of the smaller $\operatorname{arc} D_{\min }$. We need a reamer to make a cone. The main parameters of the sweep of the cone: the outer radius or the radius of the sweep $R_{R}$, the length of the outer arc $L$, the sweep angle $\alpha$ and the width of the plate $a$. 

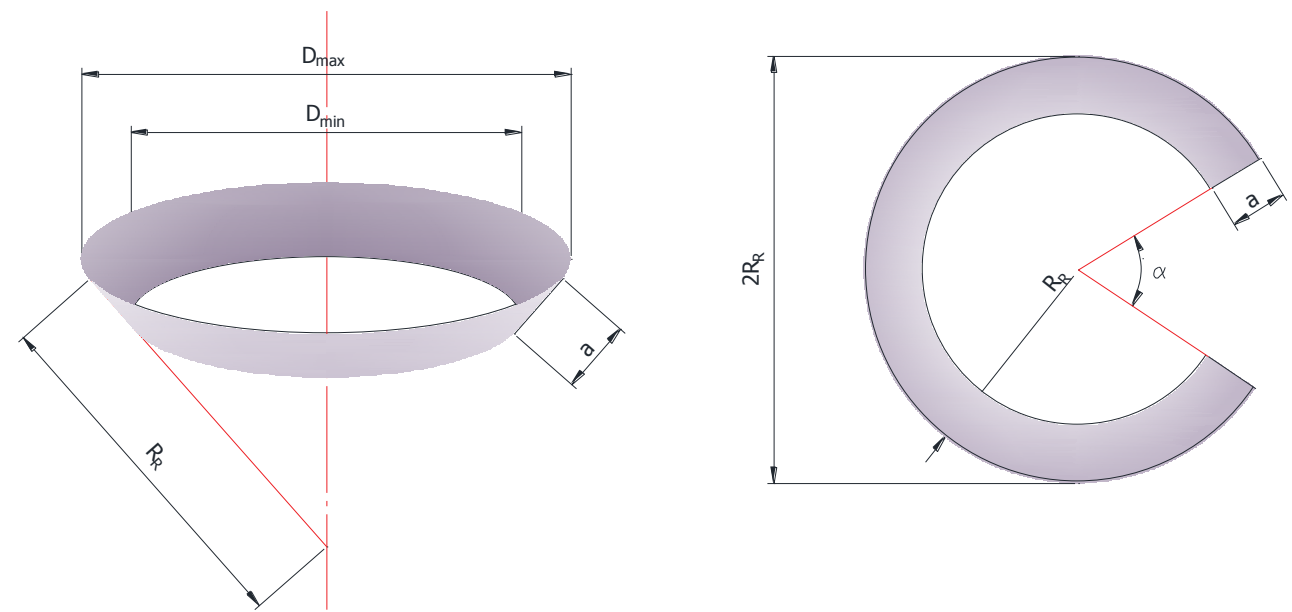

Figure 7 - Dimensions of the reflector cone of the optical system

Table 2. Calculation of the cones of the LUCH solar concentrator

\begin{tabular}{|c|c|c|c|c|c|c|c|c|c|}
\hline \multirow[t]{2}{*}{$\begin{array}{l}\ddot{E} \\
\tilde{\Xi} \\
\text { Z }\end{array}$} & $\begin{array}{c}\text { Larger } \\
\text { dia- } \\
\text { meter, } \\
\text { mm }\end{array}$ & $\begin{array}{c}\text { Smal-ler } \\
\text { diame- } \\
\text { ter, mm } \\
\end{array}$ & 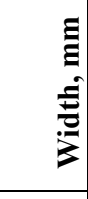 & 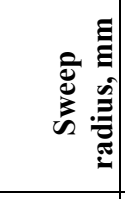 & 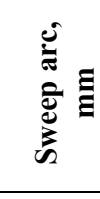 & 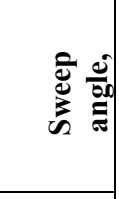 & \multirow[t]{2}{*}{$\begin{array}{l}0 \\
0 \\
\mathbb{1} \\
11 \\
0\end{array}$} & $\begin{array}{c}\text { Surface } \\
\text { area, } \mathbf{m m}^{2}\end{array}$ & \multirow[t]{2}{*}{ 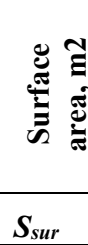 } \\
\hline & $D_{\max }$ & $D_{\min }$ & $a$ & $\boldsymbol{R}_{R}$ & $L$ & $\alpha$ & & $\boldsymbol{S}_{\text {sur }}$ & \\
\hline \multicolumn{10}{|c|}{ «Верхние» конуса } \\
\hline 1 & 2408 & 2204 & 150 & 1771 & 7565 & 115,2 & 244,8 & 1086676,9 & 1,09 \\
\hline 2 & 2204 & 2012 & 150 & 1722 & 6924 & 129,6 & 230,4 & 993371,6 & 0,99 \\
\hline 3 & 2012 & 1836 & 150 & 1715 & 6321 & 148,8 & 211,2 & 906663,6 & 0,91 \\
\hline 4 & 1836 & 1672 & 150 & 1679 & 5768 & 163,2 & 196,8 & 826553,0 & 0,83 \\
\hline 5 & 1672 & 1528 & 150 & 1742 & 5253 & 187,2 & 172,8 & 753982,2 & 0,75 \\
\hline 6 & 1528 & 1384 & 150 & 1592 & 4800 & 187,2 & 172,8 & 686123,8 & 0,69 \\
\hline 7 & 1384 & 1252 & 150 & 1573 & 4348 & 201,6 & 158,4 & 621092,9 & 0,62 \\
\hline \multicolumn{10}{|c|}{ «Нижние» конуса } \\
\hline 8 & 1260 & 1028 & 150 & 815 & 3958 & 81,6 & 278,4 & 539097,3 & 0,54 \\
\hline 9 & 1028 & 780 & 150 & 622 & 3230 & 62,4 & 297,6 & 426000,0 & 0,43 \\
\hline 10 & 792 & 516 & 150 & 430 & 2488 & 28,8 & 331,2 & 308190,2 & 0,31 \\
\hline 11 & 516 & 220 & 150 & 261 & 1621 & 4,8 & 355,2 & 173415,9 & 0,17 \\
\hline 12 & 220 & 0 & 110 & 110 & 691 & 0,0 & 360,0 & 38013,3 & 0,04 \\
\hline & & & & & & & $\sum$ & 7359180,8 & 7,36 \\
\hline
\end{tabular}

Having a certain number of cones and their main dimensions $D_{\max }$ and $D_{\min }$, one can calculate the dimensions of their sweeps. Sweep radius $R_{R}$ is determined by:

$$
R_{R}=a^{D_{\max }} / D_{\max }-D_{\min }
$$

The length of the outer $\operatorname{arc} L$ is calculated by:

$$
L=\pi \cdot D_{\max }
$$


To find the sweep angle $\alpha$, consider the ratio: the length of the outer circle $2 \pi R$ refers to the angle $360^{\circ}$ since the length of the outer arc $L$ refers to the angle $360^{\circ}-\alpha$.

After transformation we get:

$$
360^{\circ} / 360^{\circ}-\alpha=2 \pi R_{R} / L
$$

The sweep angle $\alpha$ is:

$$
360^{\circ} / 360^{\circ}-\alpha=2 R_{R} / D_{\max }
$$

$$
\alpha=360^{\circ} \cdot\left(1-D_{\max } / 2 R_{R}\right)
$$

Using the above expressions and dependences, the optical system of a solar concentrator with a diameter of 2.4 meters was calculated [6]. The results are shown in Table 2.

Possibly the best relationship between the geometric parameters of the concentrator for a given thermal power of the solar plant takes into account the coefficient $K_{x}$ (the ratio of the area of the reflective surface of the concentrator $S_{\text {sur }}$ to the area of the captured solar flux $S_{s o l}$ ). It has been experimentally established that the coefficient $K_{x}$ should be in the range from 1.3 to 1.7 .

The aperture area for the LUCH solar plant is $4.55 \mathrm{~m}^{2}$ and the total surface area of all the cones or the reflection area is $7.36 \mathrm{~m}^{2}$. The ratio of the mirror area to the aperture area is 1.62 . A high concentration accuracy on a relatively small surface of the receiver is not required as the considered concentrator in the form of cones is intended for operation in "household" solar systems (low power) used for hot water supply and heating systems. The higher the concentration factor, the higher the temperature on the surface of the receiver and for these areas of application high temperatures (above $100^{\circ} \mathrm{C}$ ) are not used. Therefore, the size of the generatrix of the solar concentrator cones can be assumed to be "relatively large", within 10-20 cm. High accuracy of the system for tracking the trajectory of the Sun is also not needed and it allows simplifying the design.

\section{CONCLUSION}

The following conclusions can be drawn analyzing the given geometric parameters of two concentrators:

1. The set goal of creating a new design of the concentrator has been achieved - to transform the paraboloid concentrator geometrically so as to simplify its design for manufacturing and operation.

2. The developed design of the LUCH solar concentrator is close to an ideal paraboloidal one, for example, the maximum deviation of the concentrator height is less than $0.1 \mathrm{~m}$ with a diameter of $2.4 \mathrm{~m}$, with equal geometrical and optical-energy parameters.

3. It is possible to estimate the degree of "illumination" of the surface of the heat receiver, its uniformity, placed at this point by the magnitude of the focal length. The larger the focus, the smaller the surface of the heat receiver perceives concentrated radiation. Conversely, the shorter the focal length, the more part of the heat sink surface is illuminated.

4. The closest coincidence of the geometrical and optical-energy parameters of the two concentrators is obtained with the same surface area of the mirror $S_{\text {sur }}$ and the coefficient $K_{x}$ which allows us to write the mathematical expression of the "LUCH" concentrator in the form of the canonical parabola equation:

$$
y^{2}=2 p x=2 \cdot 0,6428 x=1,2857 x
$$

5. A clear advantage of the developed design is the improvement of the aerodynamic properties, namely, the reduction of the wind load on the building structures (high airflow of the concentrator structure). This makes it possible to produce a concentrator with large cross-sectional dimensions and to increase the heat output of one solar plant. 


\section{Bibliography}

1. L. Li, B. Wang, J. Pottas, W. Lipiński 2019 Solar Energy 183: 805-811.

2. S.S. Rao, H.G. Lee, Y. Hu 2014 Journal of Mechanical Design, Transactions of the ASME 136(9) [091402].

3. M. Tian, Y. Su, H. Zheng, G. Pei, G. Li, S. Riffat 2018 Renewable and Sustainable Energy Reviews 82: 1272-1296.

4. S.S.S.Baljit, H.-Y.Chan, V.A. Audwinto, S.A. Hamid, A. Fudholi, S.H. Zaidi, M.Y. Othman, K. Sopiana 2017 Renewable Energy 114: Part B, 1258-1271.

5. M.R. Rodríguez-Sánchez, C. Marugán-Cruz, A. Acosta-Iborra, D. Santana 2018 Solar Energy 165: 43-54.

6. В.М. Андреев, В.А. Грилпхес, В.Д. Румянцев. Фотоэлектрическое преобразование концентрированного солнечного излучения. - Л.: Наука, 1989. -310 с.

7. $V$. Severyanin, P. Yanchilin. Bulletin of the Brest State Technical University: Water construction, heat power engineering, geoecology, 2: 74-77. (2010) (in Russian). 